\title{
MiR-203a functions as a tumor suppressor in bladder cancer by targeting SIX4
}

\author{
X. Y. NA, X. S. SHANG, Y. ZHAO, P. P. REN, X. Q. HU* \\ People's Hospital of Zhenhai District, Ningbo, Zhejiang Province, China \\ ${ }^{*}$ Correspondence: huxiaoquan@yahoo.com
}

Received May 12, 2018 / Accepted September 12, 2018

\begin{abstract}
Increasing evidence indicates that microRNAs (miRNAs) have essential roles in various biological processes, including proliferation, migration, invasion, cell cycle progression and apoptosis. It is considered that miRNA de-regulation contributes to tumor progression and metastasis in various cancers, and MiR-203a has been identified as a tumor suppressor in cancers, such as glioma, gastric cancer and hepatocellular carcinoma. Herein, we established that miR-203a expression is significantly lower in bladder cancer tissues than in adjacent normal tissues, and that low miR-203a expression is associated with poor patient outcome. The over-expression of miR-203a inhibited bladder cancer cell proliferation, invasion, migration and EMT in vitro, and its up-regulation led to bladder cancer cell cycle arrest and apoptosis. This over-expression also inhibited the PI3K/Akt signaling pathway. Bioinformatics prediction software and luciferase reporter assay then confirmed that SIX4 is a direct target of miR-203a. We established negative correlation between SIX4 expression and miR-203a expression in bladder cancer tissues, and SIX4 silencing caused effects similar to miR-203a up-regulation Furthermore, SIX4 over-expression diminished the effects of miR-203a on bladder cancer cells in vitro. In summary, our study determined that miR-203a down-regulation is closely related to tumorigenesis in bladder cancer; thus suggesting that miR-203a is a potential prognostic marker and a potential target in bladder cancer treatment.
\end{abstract}

Key words: bladder cancer, miR-203a, SIX4, PI3K/Akt, EMT

Bladder cancer is one of the most common types of cancer, and its incidence has increased in recent years [1]. Many factors contribute to the initiation and progression of bladder cancer; including aberrant cell cycle progression and excessive anti-apoptotic and pro-survival signaling [2, 3]. Despite significant progression in surgery and chemotherapy, the overall survival rate of patients with bladder cancer remains unsatisfactory. Therefore, novel predictive biomarkers and effective therapeutic strategies against bladder cancer are urgently required.

MicroRNAs (miRNAs) are endogenous, noncoding RNAs that post-transcriptionally regulate downstream gene expression by binding to complementary sequences in the 3'-UTR of their target genes $[4,5]$. Increasing evidence indicates that miRNAs actively participate in a variety of biological activities, such as cell proliferation, migration and invasion $[6,7]$, and many miRNAs have been reported to be involved in the regulation of tumorigenesis and metastasis in bladder cancer. For example, miR-137 functions as an oncogene by inhibiting the PAQR3 tumor suppressor gene in bladder cancer cells [8]. While MiR-301b negatively regulates EGR1 and thereby promotes proliferation, mobility and epithelial- to-mesenchymal transition (EMT) in bladder cancer cells (BCs) [9], miR-1-3p functions as a tumor suppressor inhibiting proliferation, invasion and migration of $\mathrm{BC}$ cells by regulating SFRP1 expression [10].

Reports established that miR-203a affects tumor biological functions, including proliferation, migration and invasion in hepatocellular carcinoma (HCC), glioma, gastric cancer and nasopharyngeal carcinoma [11-13], and it also to act as a tumor suppressor by targeting different genes in bladder cancer $[14,15]$. Herein, we determined that miR-203a is significantly down-regulated in bladder cancer tissues compared to paired adjacent normal tissues. miR-203a significantly represses proliferation, migration, invasion and EMT of bladder cancer cells. Moreover, overexpression of miR-203a lead to cell cycle arrest and apoptosis of bladder cancer cells. We then demonstrated that the SIX4 miR-203a target has an essential role in BC cell proliferation, migration, invasion, cell cycle progression and apoptosis. The combined results therefore provide new evidence that miR-203a functions as a tumor suppressor in BC cells, and it provides a novel therapeutic target in bladder cancer treatment. 


\section{Materials and methods}

Tissue samples and cell culture. A total of 40 pairs of bladder cancer tissues and adjacent normal tissues from patients undergoing resection for bladder cancer were collected and catalogued at the People's Hospital of Zhenhai, Ningbo. The study protocol was approved by the Ethics Committee of the People's Hospital of Zhenhai and written informed consent was obtained from all patients. SV-HUC-1 human uro-epithelial cells and the bladder cancer cell lines T24, EJ, J8 and 5637 were obtained from the Shanghai Bank of Cell Lines (Shanghai, China). Cells were cultured in RPMI 1640 medium (Gibco, USA) or DMEM media (Gibco) supplemented with $10 \%$ FBS (Gibco) and $1 \%$ penicillin/streptomycin. All cells were cultured in a humidified atmosphere of $5 \% \mathrm{CO}_{2}$ at $37^{\circ} \mathrm{C}$.

RT-PCR. Total RNA was purified from bladder cancer tissues and cell lines using TRIzol reagent (Life Technologies, Carlsbad, CA, USA) according to manufacturer instructions. RNA quality was determined by measuring the A260/A280 ratio by NanoDrop spectrophotometer (Thermo Fisher Scientific, USA). One microgram of RNA was reverse transcribed into cDNA using a TIANScript II cDNA First Chain Synthesis kit (Tiangen, China). Realtime PCR analysis was performed by NCode ${ }^{\mathrm{Tm}}$ SYBR Green miRNA quantitative reverse transcription PCR (RT-qPCR) kit (Thermo Fisher Scientific) and relative expression was calculated using the comparative cycle threshold $\left(2^{-\Delta \Delta \mathrm{Ct}}\right)$. The transcription level of either U6 or GAPDH was used as internal control.

Cell viability assay. Cell viability was assessed as described [16]. Briefly, cells were seeded in a 96-well plate at a density of $1 \times 10^{4}$ cells/well. Following transfection, $20 \mu \mathrm{l}$ MTT $(5 \mathrm{mg} / \mathrm{ml})$ was added to each well and $150 \mu \mathrm{l}$ dimethyl sulfoxide (DMSO) was added to each well after incubation at $37^{\circ} \mathrm{C}$ for 4 hours. Optical density at $450 \mathrm{~nm}$ was then measured by microplate reader.

Cell transfection. The miR-203a mimic (miR-203a), miRNA negative control (miR-NC), siRNA against SIX4 (si-SIX4) and siRNA negative control (si-NC) were purchased from Genepharm (Suzhou, China) and transfected into cells with Lipofectamine 2000 (Life Technologies). For the transient expression of SIX4, cells were transfected with the pCMV-SIX4 plasmid containing SIX4 or with an empty vector control (created by RyoBio Company, China) using Lipofectamine 2000. The transfection efficiency was confirmed by Western blotting.

Dual-luciferase reporter assay. Two oligonucleotide pairs containing either a mutant or wild-type SIX4 3'-UTR sequence were synthesized by Genepharm (Suzhou, China). After annealing, they were inserted into the pmirGLO vector (Promega, USA). For the luciferase assays, cells were co-transfected with the corresponding vectors and the miR-203a mimic with Lipofectamine 2000 (Life Technologies) Twentyfour hours after transfection, the firefly and Renilla luciferase activities were consecutively detected by dual-luciferase kit (Promega Corporation), and the relative luciferase activity was normalized to the Renilla luciferase activity.

Wound healing assay. After transfection, cells were grown to confluence in six-well plates. A $200 \mu \mathrm{l}$ micropipette tip made a cross wound, and wound healing was observed $24 \mathrm{~h}$ later. Images were acquired by phase-contrast microscopy (Olympus, Japan) immediately after or $24 \mathrm{~h}$ after wounding.

Cell invasion assay. The cell invasion assay was conducted using Transwell chambers (Millipore, USA). Transwell chamber inserts were placed in a 24-well plate. The inserts were then coated with Matrigel (BD Bioscience, USA) on the upper surface and incubated for $30 \mathrm{~min}$ at $37^{\circ} \mathrm{C}$. After transfection, $8 \times 10^{4}$ cells were suspended in $200 \mu \mathrm{l}$ serumfree medium and added to the upper chambers and $200 \mu \mathrm{l}$ medium supplemented with $10 \%$ FBS was added to the lower chambers as chemo-attractant. After incubation at $37^{\circ} \mathrm{C}$ for $24 \mathrm{~h}$, the cells on the upper surface of the membrane were carefully removed using a cotton swab. The cells that crossed the Matrigel membrane were fixed with $100 \%$ methanol and stained with $0.1 \%$ crystal violet. Ten random visual fields were selected for each insert, and the cells were counted $\times 200$ under a light microscope (OlympUSA)

Cell cycle analysis. After transfection for $24 \mathrm{~h}$, cells were fixed with $75 \%$ ethanol at $-20^{\circ} \mathrm{C}$ overnight and then stained with a propidium iodide (PI)/RNase staining buffer (Sigma, USA) for 15 minutes at room temperature and subjected to flow cytometric analysis.

Apoptosis assay. As previously described [17], apoptosis rates were assayed by nucleosome ELISA (Roche, Germany). Briefly, after transfection for $24 \mathrm{~h}$, apoptosis induction was assessed by measuring the enrichment of nucleosomes in the cytoplasm, and the apoptotic rate was determined as described in manufacturer instructions.

Caspase-3/7 activity assay. The activity of caspase- $3 / 7$ was assayed using a Caspase-Glo 3/7 Assay kit (Promega, USA). After transfection for $48 \mathrm{~h}, 100 \mu \mathrm{l}$ of caspase-3/7 reagent was added to each well and incubated for $1 \mathrm{~h}$ at room temperature. The luminescence was measured using a BioTek 312e microplate reader (BioTek Instruments, USA). The caspase$3 / 7$ activity was indicated as the percentage of luminescence in untreated control wells.

Western blot assay. Cell lysates were prepared after 48-hour transfection by radioimmunoprecipitation assay (RIPA) lysis buffer (Beyotime, China). The protein concentrations were measured by Bradford assay kit (Beyotime, China). Total protein was separated by $12 \%$ SDS-PAGE and transferred to PVDF membranes (Millipore, USA) which were then blocked in 5\% skim milk at room temperature for $1 \mathrm{~h}$. After washing with TBST, membranes were incubated with primary antibodies overnight at $4{ }^{\circ} \mathrm{C}$. The following primary antibodies were used: anti-CDK1 (CST, Danvers, USA), anti-cyclin A (CST), anti-cyclin B (CST), anti-caspase-3 (CST), anti-Bcl-2 (CST), anti-Mcl-1 (CST), 
anti-E-cadherin (CST), anti-N-cadherin (CST), antivimentin (CST), anti-Snail (CST), anti-p-PI3K (CST), antiPI3K (CST), anti-p-Akt (CST), anti-Akt (CST), anti-SIX4 (Abcam, USA), and anti-GAPDH (Sigma, USA). Following three TBST washes of $15 \mathrm{~min}$ each, membranes were incubated with a goat anti-rabbit horseradish peroxidaseconjugated secondary antibody for $1 \mathrm{~h}$ at room temperature. The results were visualized using a Super Signal chemiluminescence substrate (Pierce, USA) according to manufacturer instructions.

Statistical analysis. Continuous variables are presented as mean \pm standard deviation (SDs). Student's t test compared the two experimental groups and One-way ANOVA was performed for multiple comparisons. Kaplan-Meier survival curves and log-rank tests estimated patient survival and the differences between groups. SPSS 10.0 and GraphPad Prism 5.0 (GraphPad Software, USA) was used in statistical analysis and $\mathrm{p}<0.05$ was considered statistically significant.
Results

MiR-203a is down-regulated in bladder cancer tissues and cell lines. To evaluate the expression level of miR-203a in bladder cancer tissues, quantitative real-time PCR (qRT-PCR) was performed on 40 pairs of bladder cancer tissues and adjacent normal tissues. The expression of miR-203a was significantly lower in tumor tissues than in normal tissues (Figure 1A). The miR-203a level was then detected in normal cell line SV-HUC-1 and human bladder cancer cell lines T-24, EJ, J8 and 5637. We found that the expression level of miR-203a was significantly lower in the bladder cancer cells than in SV-HUC-1 cells (Figure 1B). These results suggest that miR-203a was down-regulated in both bladder cancer tissues and cell lines.

We then investigated the relationship between miR-203a expression and different clinical parameters. The median miR-203a expression value divided the patients into high-
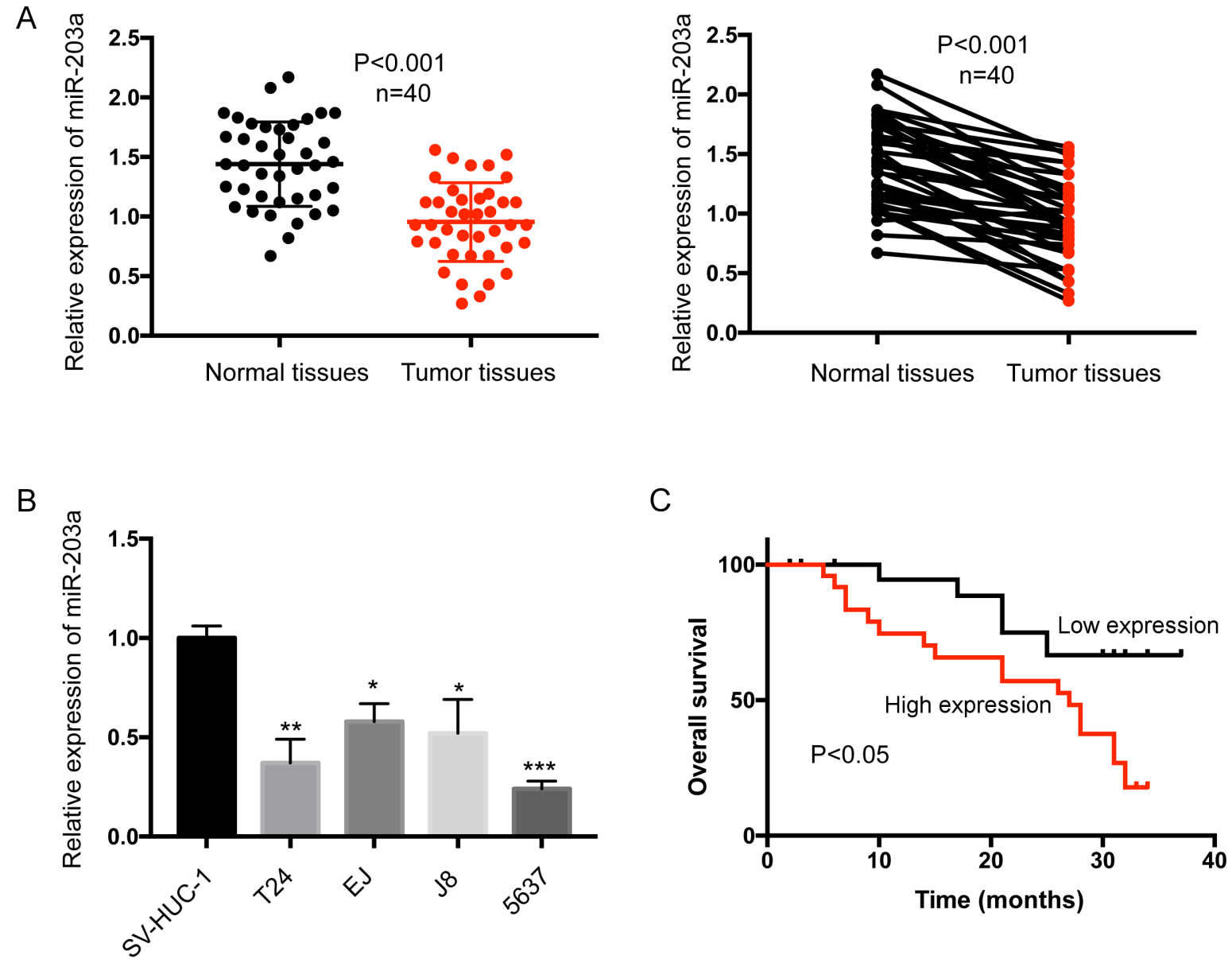

Figure 1. MiR-203a expression is down-regulated in bladder cancer tissues and cell lines and correlates with patient survival. A) MiR-203a expression in 40 pairs of bladder tissues and adjacent normal tissues was analyzed by RT-PCR; B) MiR-203a expression in uroepithelial cells (SV-HUC-1) and bladder cancer cell lines (T24, EJ, J8 and 5637) was analyzed by RT-PCR; C) Correlation between miR-203a expression and bladder cancer patient survival. ${ }^{*} \mathrm{p}<0.05,{ }^{*} \mathrm{p}<0.01$; the data is in means \pm standard deviations (SDs) of triplicate measurements. 
expression $(\mathrm{n}=20)$ and low-expression $(\mathrm{n}=20)$ groups. Table 1 highlights that miR-203a was closely associated with tumor stage $(\mathrm{p}=0.025)$ and lymph node metastasis $(\mathrm{p}=0.01)$. However, there was no significant correlation between miR-203a expression and other clinical-pathological factors; including gender, age, tumor size and histological grade. The Kaplan-Meier analysis of the 40-month follow-up survival survey revealed that low expression of miR-203a predicated poor survival in patients with bladder cancer $(\mathrm{p}<0.05)$ (Figure $1 \mathrm{C})$. These findings suggest that the aberrant expression of miR-203a is involved in tumorigenesis and bladder cancer development.

miR-203a effects on bladder cancer cell proliferation, migration and invasion. To discover the role of miR-203a in bladder cancer tumorigenesis, we transfected T24 and 5637 cells with the miR-203a mimic, and the qRT-PCR results indicate that miR-203a expression is significantly up-regulated in bladder cancer cells after transfection with the miR-203a mimic but not the miR-NC mimic (Figure 2A). The MTT assay revealed that miR-203a over-expression significantly inhibited bladder cancer cell proliferation (Figure 2B). We then studied miR-203a

Table 1. Clinical characteristics and miR-203a expression levels of patients with bladder cancer.

\begin{tabular}{|c|c|c|c|c|}
\hline \multirow{2}{*}{ Variables } & \multirow{2}{*}{ Total } & \multicolumn{2}{|c|}{$\begin{array}{c}\text { Expression of } \\
\text { miR-203a }\end{array}$} & \multirow{2}{*}{ p-value } \\
\hline & & $\begin{array}{c}\text { Low } \\
(n=20)\end{array}$ & $\begin{array}{c}\text { High } \\
(n=20)\end{array}$ & \\
\hline \multicolumn{5}{|l|}{ Age (years) } \\
\hline$<60$ & 15 & 7 & 8 & 0.744 \\
\hline$\geq 60$ & 25 & 13 & 12 & \\
\hline \multicolumn{5}{|l|}{ Gender } \\
\hline Female & 17 & 10 & 7 & 0.337 \\
\hline Male & 23 & 10 & 13 & \\
\hline \multicolumn{5}{|c|}{ Tumor Stage } \\
\hline $\mathrm{T} 1-\mathrm{T} 2$ & 17 & 5 & 12 & 0.025 \\
\hline T3-T4 & 23 & 15 & 8 & \\
\hline \multicolumn{5}{|c|}{ Tumor Size } \\
\hline$<5 \mathrm{~cm}$ & 25 & 11 & 14 & 0.327 \\
\hline$\geq 5 \mathrm{~cm}$ & 15 & 9 & 6 & \\
\hline \multicolumn{5}{|c|}{ Lymph node metastasis } \\
\hline Yes & 16 & 12 & 4 & 0.010 \\
\hline No & 24 & 8 & 16 & \\
\hline \multicolumn{5}{|c|}{ Histological grade } \\
\hline G1 & 13 & 7 & 6 & 0.736 \\
\hline G2, G3 & 27 & 13 & 14 & \\
\hline
\end{tabular}

A

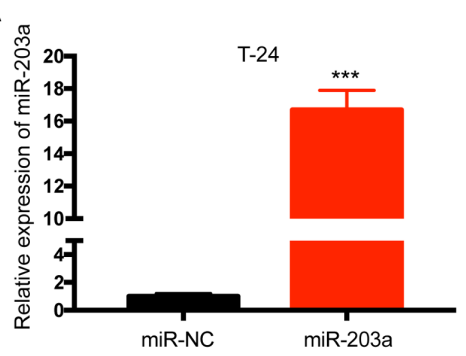

B

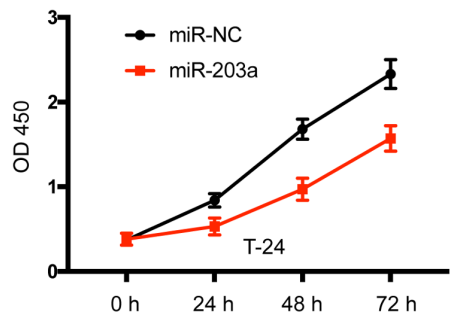

C
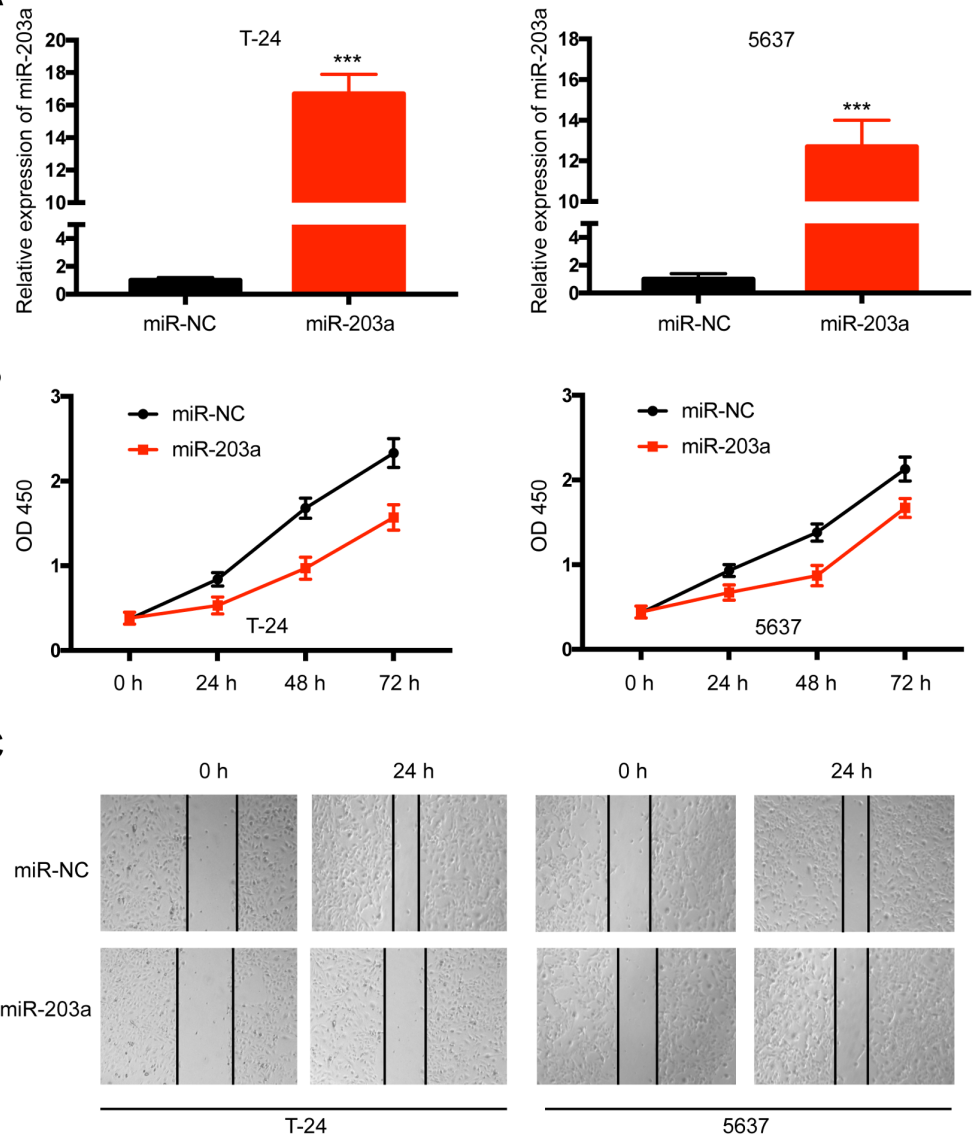

$\mathrm{Oh}$
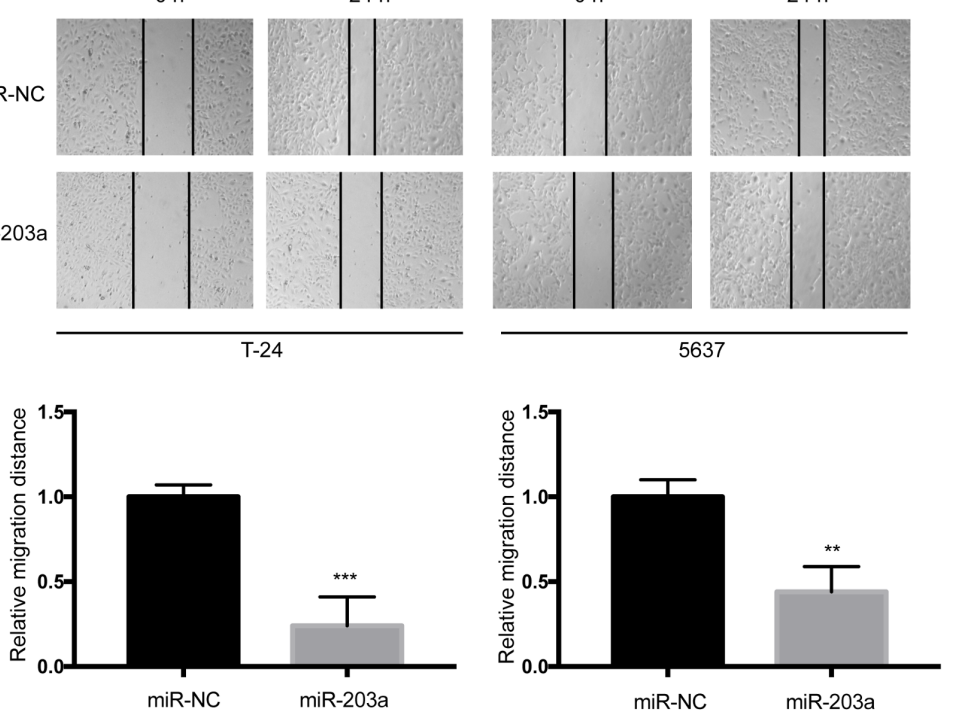

D
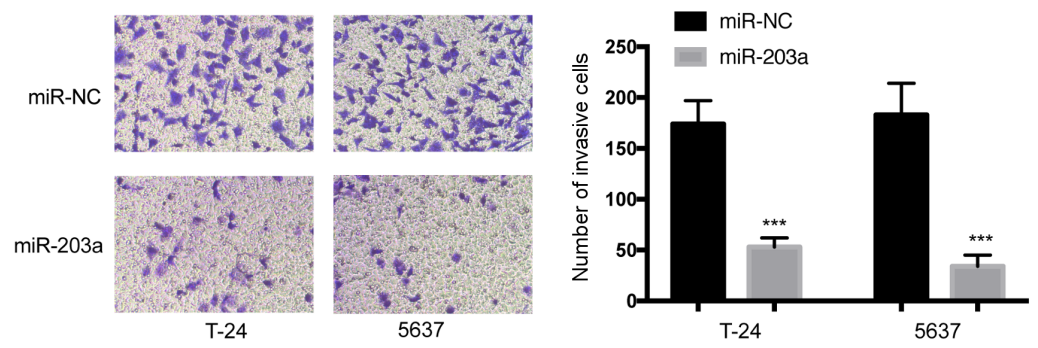

Figure 2. Over-expression of miR-203a represses the proliferation, migration and invasion of bladder cancer cells. A) After transfection with the miR-203a mimic or miR-NC for $24 \mathrm{~h}$, the expression of miR-203a in T24 and 5637 cells was analyzed by real-time PCR; B) Influence of miR-203a over-expression on the proliferation of T24 and 5637 cells by an MTT assay; C) Wound healing assay of T24 and 5637 cells after transfection with miR-203a or miR-NC mimics; D) Invasion assay of T24 and 5637 cells after transfection with an miR-203a mimic. ${ }^{* *} p<0.01$, ${ }^{* *} \mathrm{p}<0.001$; the data is in means \pm standard deviations (SDs) of triplicate measurements. 
A

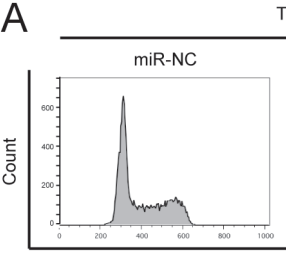

$\mathrm{T}-24$

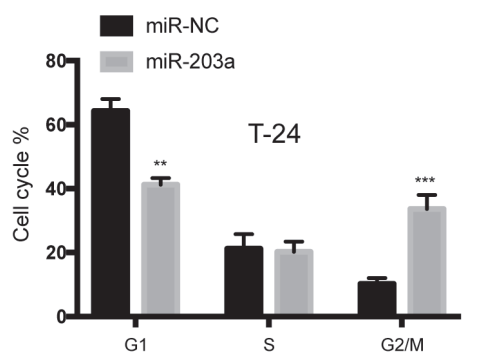

B

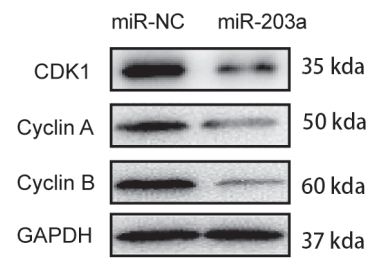

$\mathrm{T}-24$

C

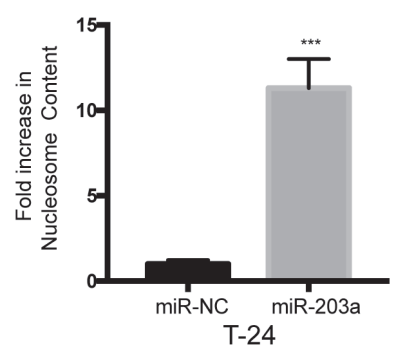

D

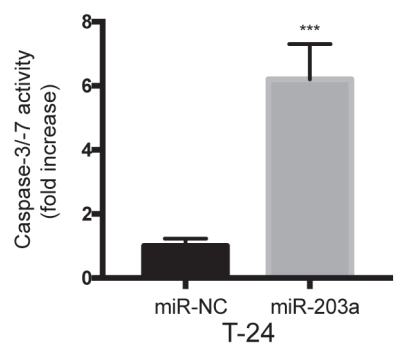

E

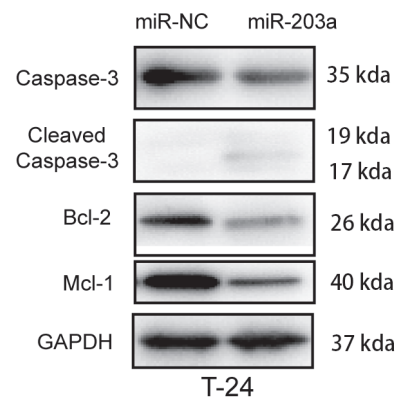

5637

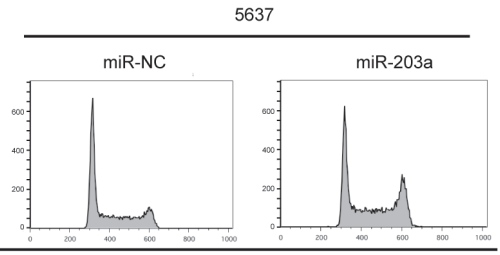

DNA content
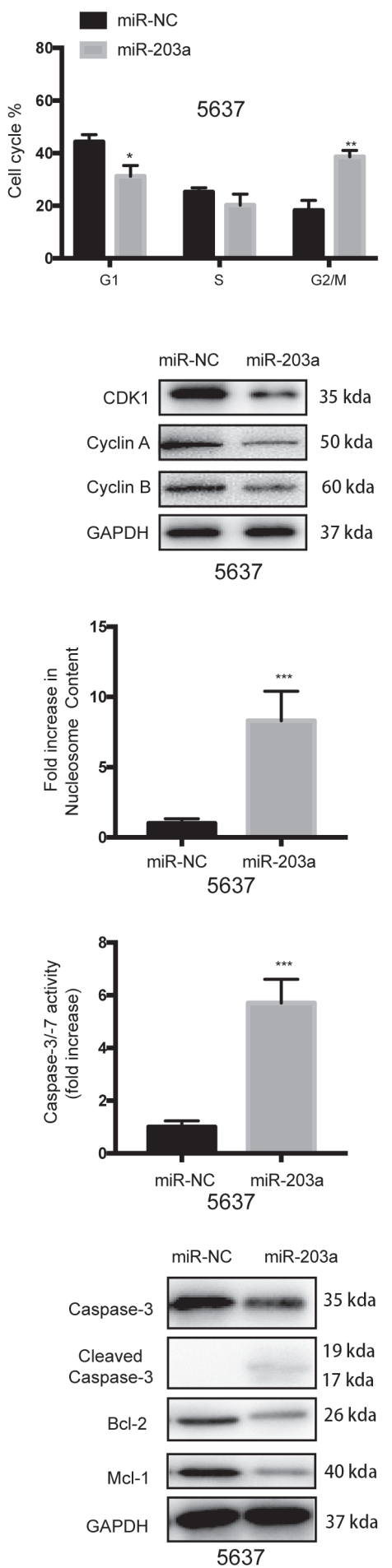

effect on bladder cancer cell migration and invasion. Figure $2 \mathrm{C}$ shows that miR-203a over-expression significantly inhibited the migration of both T24 and 5637 cells in the wound healing assay and dramatically repressed the invasive ability of bladder cancer cell invasive ability (Figure 2D). Results indicate that miR-203a significantly inhibits bladder cancer cell proliferation, migration and invasion.

miR-203a effect on bladder cancer cell-cycle progression and apoptosis. To investigate the mechanisms underlying miR-203a-mediated cell growth inhibition, flow cytometry analyzed the distribution of the cell cycle in T-24 and 5637 cells after transfection with miR-203a. This revealed statistically significant G2/M arrest in both T24 and 5637 cells (Figure 3A). We then analyzed the expression of cell cycle-related proteins by Western blotting. As shown in Figure 3B, the over-expression of miR-203a led to down-regulation of CDK1, cyclin A and cyclin $\mathrm{B}$, so we investigated whether miR-203a also affects bladder cancer cell apoptosis The nucleosome ELISA indicated that miR-203a over-expression resulted in greater bladder cancer cell apoptosis than miR-NC over-expression (Figure 3C). To further confirm the effects of miR-203a on the apoptosis of bladder cancer cells, caspase-3/7 activity assay was performed, and this revealed that the over-expression of miR-203a caused greater activation of caspase-3/7 than the over-expression of miR-NC (Figure 3D). Western blotting confirmed that miR-203a over-expression led to both caspase- 3 cleavage and the down-regulation of the Bcl-2 and Mcl-1 proteins which are vital regulators of apoptosis (Figure 3E). This indicates that miR-203a over-expression caused apoptosis and cell cycle arrest at the G2/M phase.

Figure 3. Over-expression of miR-203a induces cell cycle arrest and apoptosis in bladder cancer cells. A) After transfection with the miR-203a mimic or miR$\mathrm{NC}$ for $48 \mathrm{~h}$, cells were stained with propidium iodide and analyzed by flow cytometry; B) After transfection for $48 \mathrm{~h}$, cell lysates were subjected to Western blot analysis with the indicated antibodies; $\mathrm{C}$ ) After transfection for $48 \mathrm{~h}$, cell apoptosis rates were assessed; D) After transfection for $48 \mathrm{~h}$, caspase-3/7 activity was assessed; E) Cell lysates were subjected to a western blot analysis with the indicated antibodies. ${ }^{*} \mathrm{p}<0.05$, ${ }^{* *} \mathrm{p}<0.01,{ }^{* * *} \mathrm{p}<0.001$; the data is in means \pm standard deviations (SDs) of triplicate measurements. 
A

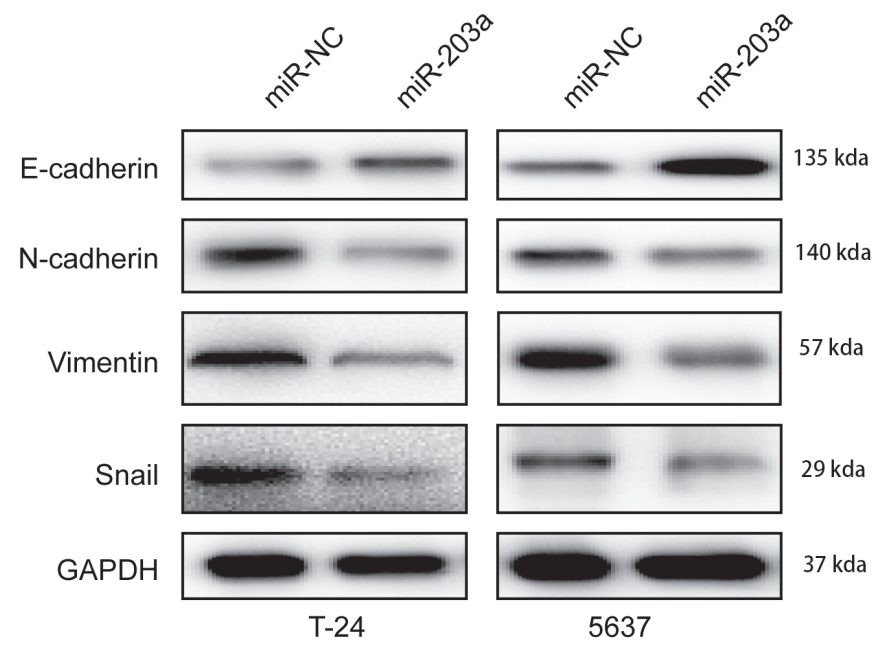

B

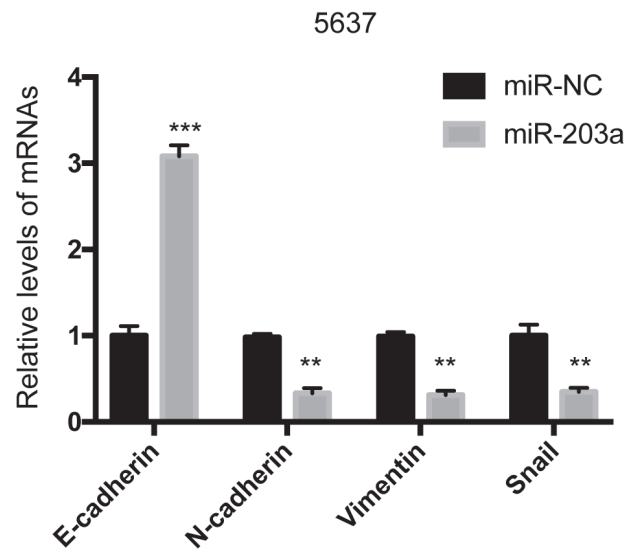

C

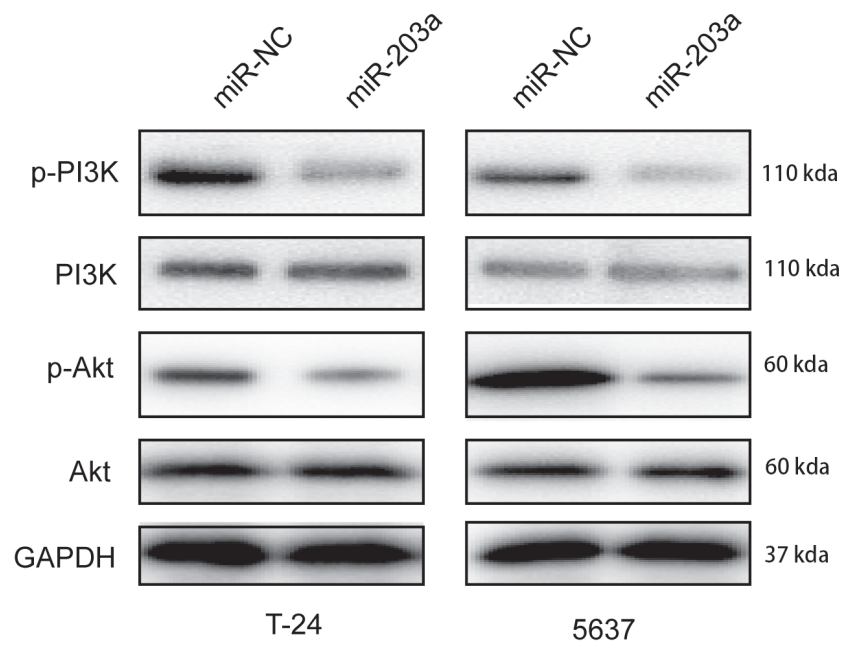

Figure 4. miR-203a inhibits EMT and the PI3K/Akt signaling pathway in bladder cancer cells. Bladder cancer cells were transfected with miR-NC or the miR-203a mimic for $48 \mathrm{~h}$. A) Cell lysates were subjected to a western blot analysis with the indicated antibodies; B) E-cadherin, N-cadherin, vimentin and Snail mRNA levels were analyzed by RT-PCR; C) PI3K/Akt signaling pathway proteins were analyzed by western blotting. ${ }^{* *} \mathrm{p}<0.01$; the data represent the means \pm standard deviations (SDs) of triplicate measurements. 
MiR-203a suppresses EMT progression and the PI3K/ Akt signaling pathway in vitro. To test the effect of miR-203a on EMT progression in bladder cancer cells, the protein levels of EMT markers were analyzed by western blotting. The forced expression of miR-203a increased the expression of the epithelial marker protein E-cadherin but decreased the expression of the $\mathrm{N}$-cadherin, vimentin and Snail mesenchymal marker proteins (Figure 4A). The RT-PCR results were consistent with the Western blotting results (Figure 4B), and miR-203a over-expression suppressed PI3K and Akt phosphorylation (Figure 4C). The combined results indicate that miR-203a suppresses both the EMT phenotype and the PI3K/Akt signaling pathway in bladder cancer cells in vitro.
SIX4 is a direct target of miR-203a in bladder cancer cells. To identify potential targets of miR-203a that contribute to bladder cancer tumorigenesis, we used miRNA target databases, including TargetScan and starBase, and found that SIX 4 was one of the predicted targets. A putative and evolutionarily conserved miR-203a binding site was in the 3'-UTR of SIX4 mRNA (Figure 5A). To confirm that SIX4 is a direct target of miR-203a, the 3'-UTR of SIX4 was cloned into a pmirGLO dual-luciferase miRNA target expression vector. In addition, a second vector was constructed in which the putative binding site of miR-203a in the 3'-UTR was mutated (Figure 5A). The results showed that the co-transfection of the SIX4 wt 3'-UTR vector and the miR-203a mimic into
A

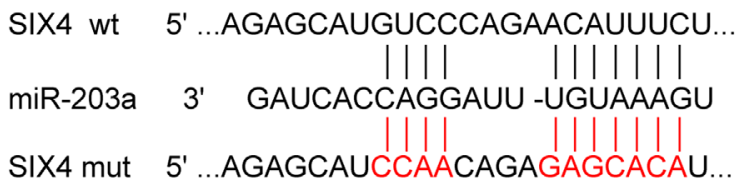

B

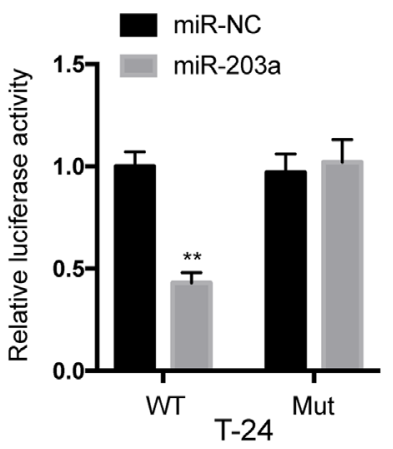

D

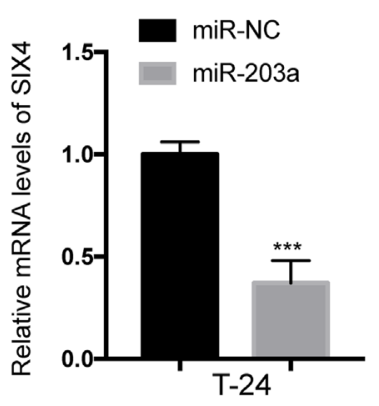

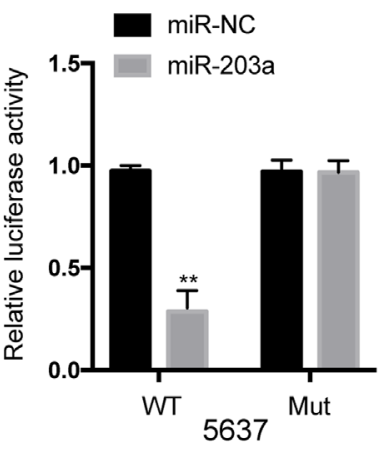

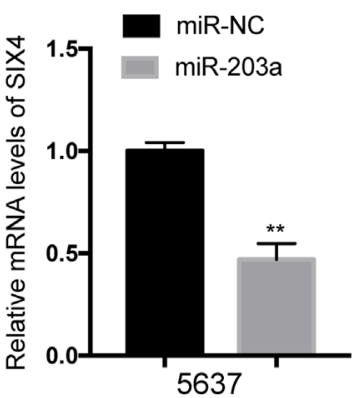

C
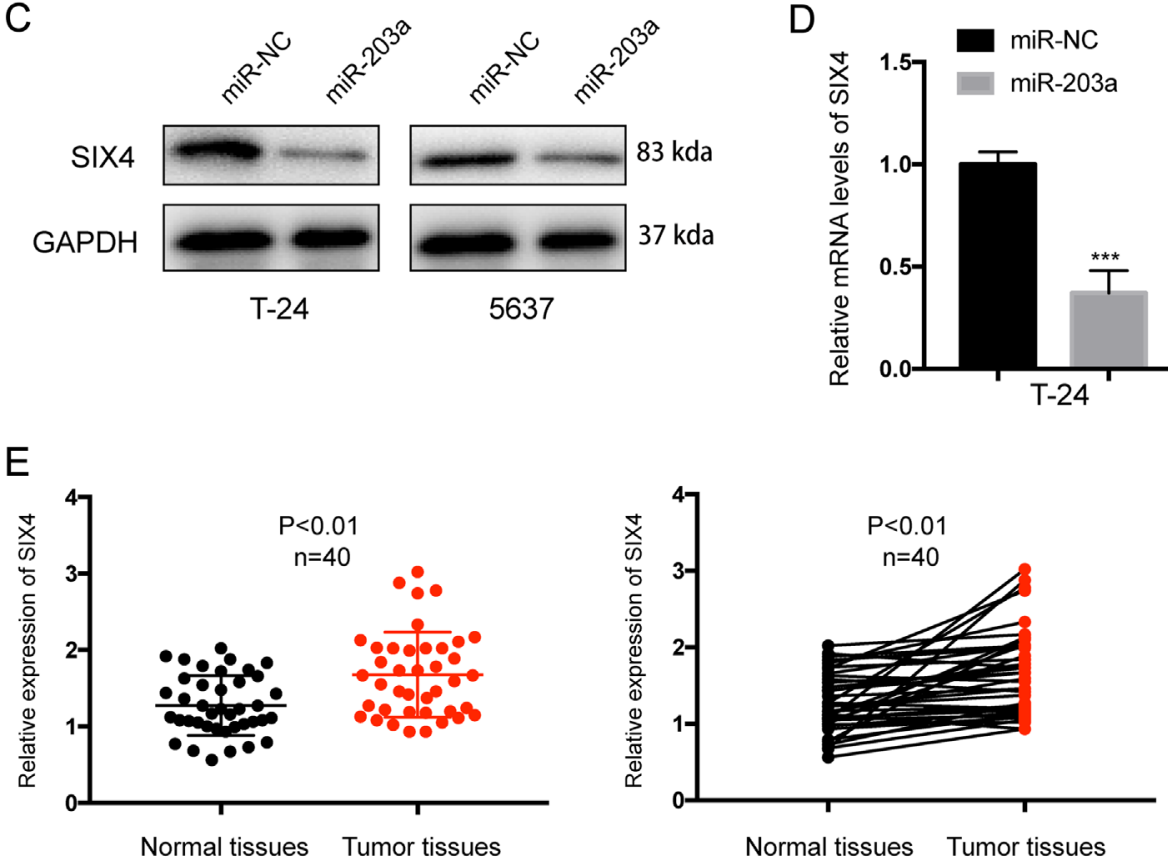

F

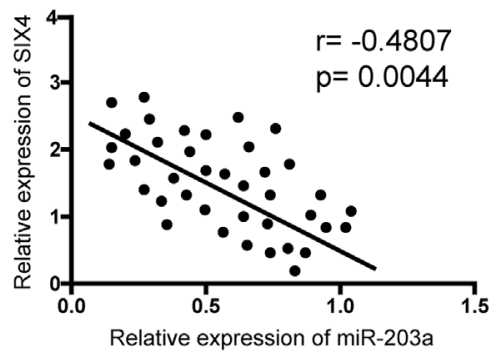

Figure 5. SIX4 is a direct target of miR-203a. A) The predicted miR-203a binding site in the SIX4 mRNA 3'-UTR and the corresponding mutation in the SIX4 3'-UTR; B) Luciferase activities of the wild-type and mutant pmir-SIX4 3'-UTR reporters in T-24 and 5637 cells; C) Western blot analysis of the SIX4 protein level after transfection with miR-NC or the miR-203a mimic for $24 \mathrm{~h}$ in T-24 and 5637 cells; D) RT-PCR analysis of SIX4 mRNA expression after transfection with miR-NC or the miR-203a mimic for $24 \mathrm{~h}$ in T-24 and 5637 cells; E) RT-PCR analysis of the SIX4 mRNA level in 40 pairs of bladder cancer and adjacent normal tissues; F) Spearman's correlation analysis was performed to analyze the association between the expression levels of miR-203a and SIX4 in bladder cancer tissues. ${ }^{* *} \mathrm{p}<0.01,{ }^{* * *} \mathrm{p}<0.01$; the data is in means \pm standard deviations (SDs) of triplicate measurements. 


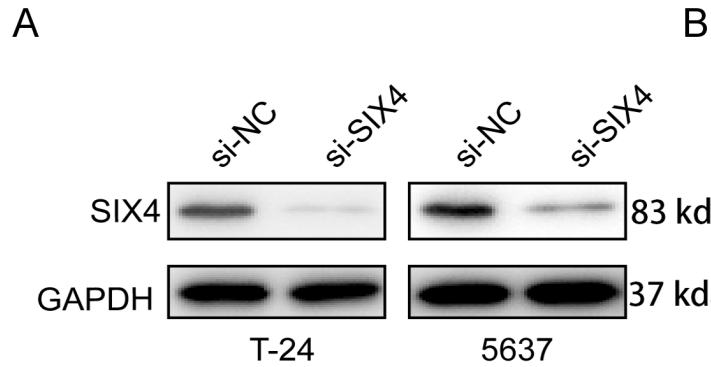

$\mathrm{T}-24$
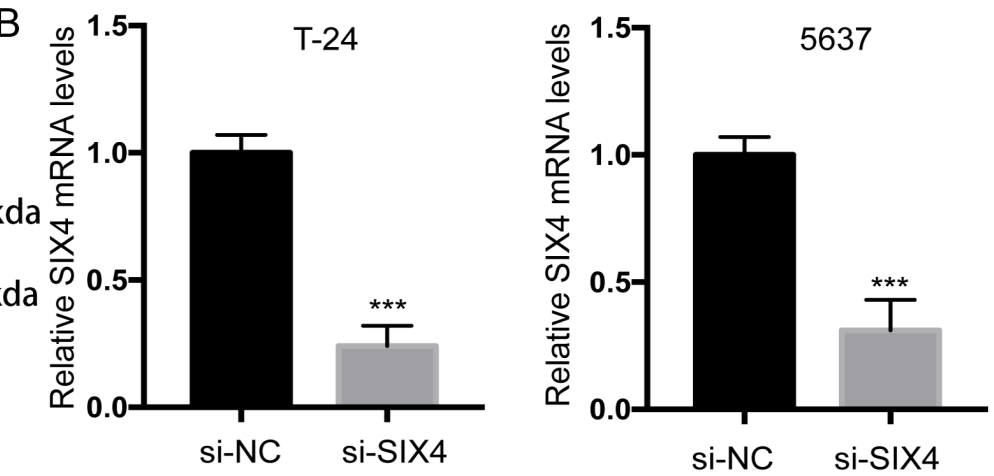

C
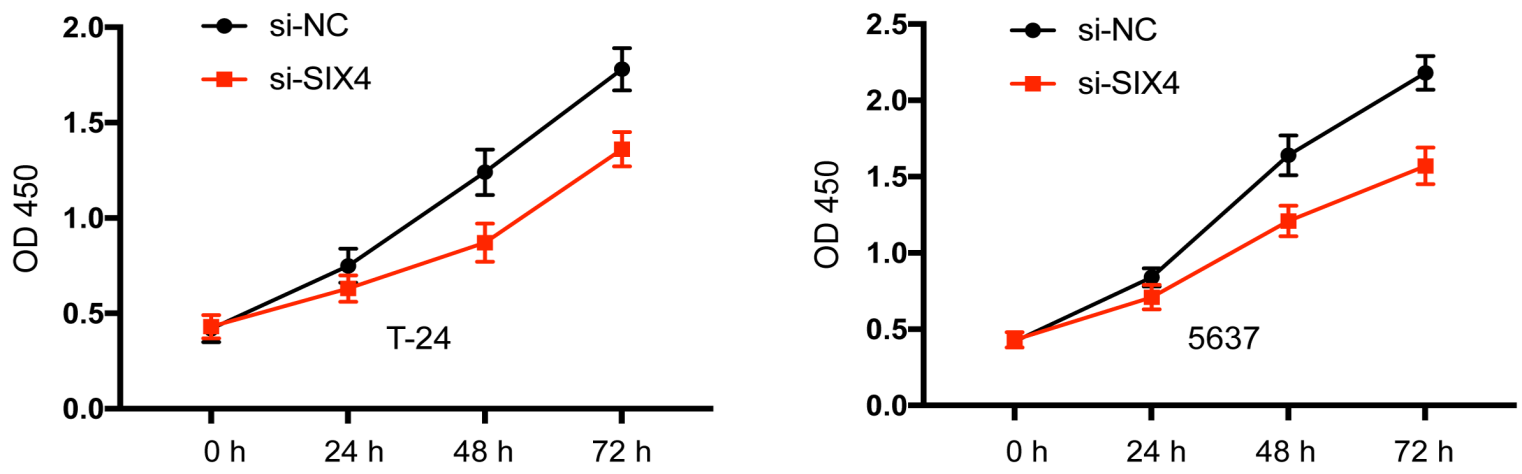

D

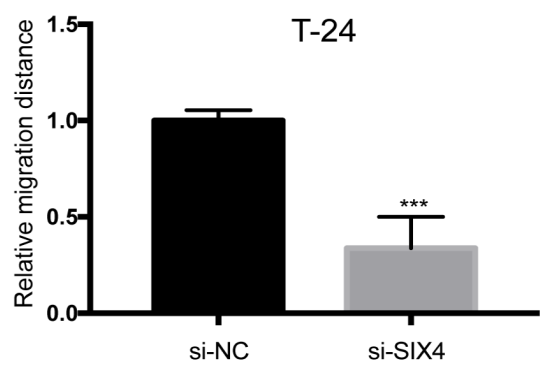

F

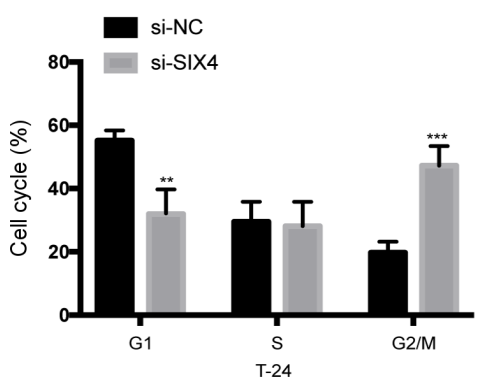

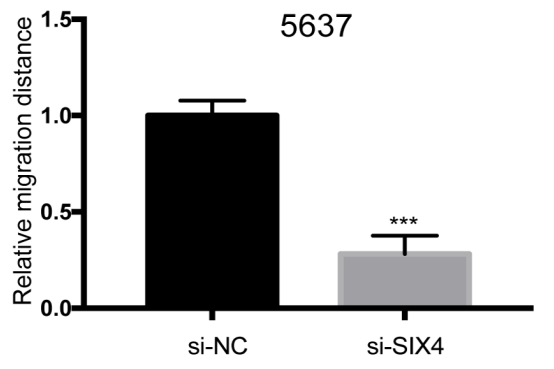

E

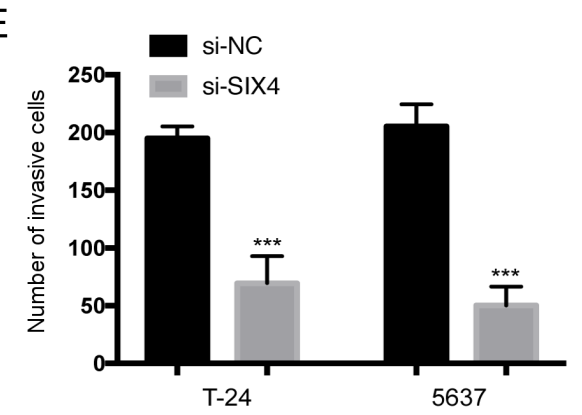

G

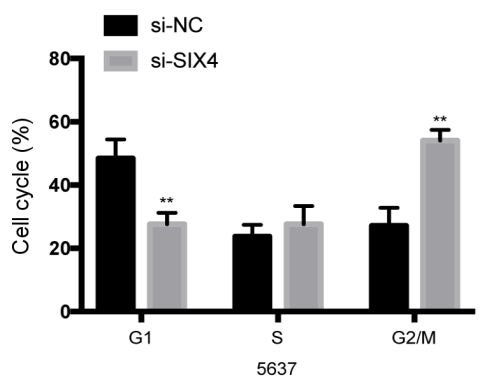

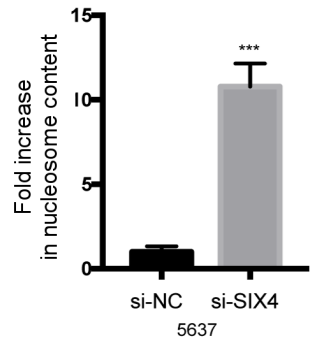

Figure 6. Silencing of SIX4 has similar effect to miR-203a transfection in bladder cancer cells. A) After transfection with si-SIX4 or si-NC for $24 \mathrm{~h}$, the protein level of SIX4 was measured by western blotting; B) After transfection with si-SIX4 or si-NC for $24 \mathrm{~h}$, the mRNA level of SIX4 was measured by RT-PCR; C) The knockdown of SIX4 suppresses the proliferation of bladder cancer cells; D) The knockdown of SIX4 suppresses the migration of bladder cancer cells; E) The knockdown of SIX4 suppresses the invasion of bladder cancer cells; F) The knockdown of SIX4 induces cell cycle arrest at the G2/M phase in bladder cancer cells; G) The knockdown of SIX4 induces apoptosis in bladder cancer cells. ${ }^{* *} \mathrm{p}<0.01,{ }^{* * *} \mathrm{p}<0.01$; the data represent the means \pm standard deviations (SDs) of triplicate measurements. 
T24 and 5637 cells significantly suppressed luciferase activity. However, co-transfection with the SIX4 mut 3'-UTR vector and the miR-203a mimic had little effect on luciferase activity in BC cells (Figure 5B). Furthermore, the Western blot and RT-PCR analyses revealed that the relative protein and mRNA expression levels of SIX4 were significantly decreased after transfection with miR-203a (Figures 5C, D). We then evaluated the mRNA level of SIX4 in bladder cancer tissues and adjacent normal tissues. Figure 5E highlights that the expression of SIX4 was significantly higher in bladder tumor tissues than in adjacent normal tissues. Correlation analysis also revealed that the expression of miR-203a negatively correlated with SIX4 mRNA level (Figure 5F). These combined results suggest that SIX4 is a direct target of miR-203a in bladder cancer cells.

Knockdown of SIX4 has a similar effect to miR-203a transfection in BC cells. To investigate the role of SIX4 in bladder cancer tumorigenesis, we used siRNA to silence SIX4. As shown in Figures 6A and B, the protein and mRNA levels of SIX 4 were successfully decreased by si-SIX 4 but not by si-NC. The knockdown of SIX4 by si-SIX4 suppressed cell viability in both bladder cancer cell lines at different time points (Figure 6C). The migration and invasion assays also showed that SIX 4 down-regulation inhibited the migration and invasion of bladder cancer cells (Figures 6D, E). The results also indicate that si-SIX 4 can induce G2/M phase arrest, leading to a significant increase in the proportion of cells in the G2/M phase. Moreover, apoptotic analysis determined that si-SIX4 had an effect similar to that of transfection with miR-203a; si-SIX4 induced more apoptosis than the si-NC control. This infers that SIX4 knockdown mimics the miR-203a biological effect.

SIX4 over-expression inhibits miR-203a effect on BC cells. To further examine the correlation between SIX4 and miR-203a, we over-expressed SIX4 in bladder cancer cells. Figures 7A and B show that both the protein and mRNA SIX4 expression were significantly up-regulated in response to SIX4 over-expression and this markedly abrogated the miR-203a inhibitory effect on T24 and 5637 cell viability (Figure 7C).
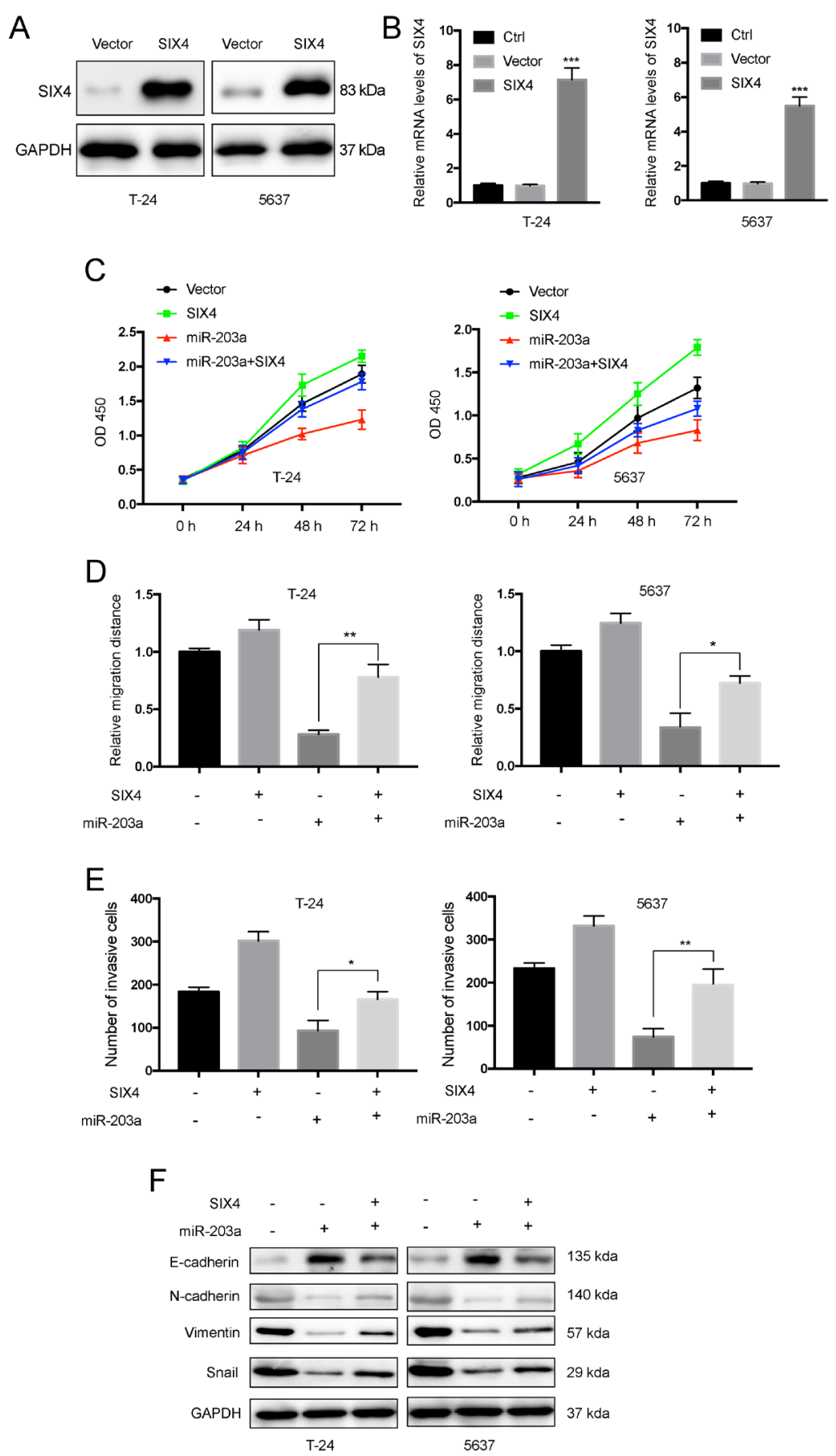

Figure 7. Overexpression of SIX4 abrogated the effects of miR-203a in bladder cancer cells. A) Bladder cancer cells were transfected with empty vector or the SIX4 expression vector for $24 \mathrm{~h}$, and the protein level of SIX4 was then measured by western blotting; B) Bladder cancer cells were transfected with empty vector or with the SIX4 expression vector for $24 \mathrm{~h}$, and the mRNA level of SIX4 was then measured by RT-PCR; C) Bladder cancer cells were transfected with empty vector, miR-203a mimic, SIX4, or the combination of miR-203a and SIX4 for the indicated durations, and cell viability was then assessed; D and E) Bladder cancer cells were transfected with empty vector, miR-203a mimic, SIX4, or the combination of miR-203a and SIX4 for $24 \mathrm{~h}$, and cell migration and invasion were then assessed. F) Bladder cancer cells were transfected with empty vector, miR-203a mimic, SIX4, or the combination of miR-203a and SIX4 for $24 \mathrm{~h}$, and total cell lysates were then subjected to Western blot with the indicated antibodies. ${ }^{*} \mathrm{p}<0.05,{ }^{* *} \mathrm{p}<0.01,{ }^{* * *} \mathrm{p}<0.01$; the data is in means \pm standard deviations (SDs) of triplicate measurements. 
Interestingly, SIX4 over-expression enhanced T24 and 5637 migration and invasion and attenuated the inhibitory effects of miR-203a on the migration and invasion of 5637 cells (Figures 7D, E). Moreover, the up-regulation of SIX4 suppressed the inhibitory effects of miR-203a on EMT in T24 and 5637 cells. These combined results further confirm SIX4 is a direct miR-203a target.

\section{Discussion}

Herein, we provide novel insight into the molecular effect of miR-203a in bladder cancer by negative regulation of SIX4 expression. Increasing evidence indicates that miRNAs have vital roles in carcinogenesis and that certain miRNAs are associated with clinical features and outcomes. Many studies have investigated the role of miRNAs in bladder cancer. A very recent report notes that miR-22 suppresses EMT in bladder cancer by dual inhibition of Snail and the MAPK signaling network [18]. Yang et al also recorded that miR-149-3p suppresses these cells' proliferation, migration and invasion by inhibition of S100A4 [19]. In addition, Feng et al reported that miR-556-3p promotes tumorigenesis in bladder cancer through DAB2IP repression [20]. It was also found that miR-294 promotes bladder cancer cell proliferation and motility by modulating the PI3K/Akt and JAK/ STAT pathways, [21].

Herein, miR-203a expression was significantly downregulated and exerted anti-tumor effect on bladder cancer tissues and cell lines compared to their normal counterparts; and previous investigations support that miR-203a expression is reduced in other cancers. For example, miR-203a is down-regulated and contributes to cell invasion in gastric cardia adenocarcinoma [22], it suppresses proliferation and metastasis in nasopharyngeal carcinoma by inhibiting LASP1 [13] and it is a confirmed tumor suppressor in bladder cancer $[14,15]$.

In contrast, however, miR-203a is significantly overexpressed in breast cancer tissues compared to normal adjacent tissues, but its down-regulation is associated with poor clinical outcome [23] and miR-203a also promotes cell proliferation and migration in renal cell carcinoma and functions there as an oncogene [24].

These discrepancies may be due to miRNAs regulating the expression of different genes in a different manner and depends on disease and cellular context. Therefore, investigation into miR-203a role in more types of cancer is warranted.

Herein, miR-203a expression was down-regulated in bladder cancer tissues and cells, and the forced expression of miR-203a inhibited cell proliferation, migration and invasion. This supports its function as a tumor suppressor in hepatocellular carcinoma, gastric carcinoma, glioma and nasopharyngeal carcinoma $[12,22,25,13]$. We also found that miR-203a induced cell cycle arrest in the G2/M phase, and this was accompanied by down-regulation of $\mathrm{CDK} 1$, cyclin $\mathrm{A}$ and cyclin B. In addition, its over-expres- sion increased E-cadherin expression but decreased that of $\mathrm{N}$-cadherin, vimentin and Snail. These results indicate that miR-203a suppressed the EMT phenotype in bladder cancer cells, and agrees with findings that it inhibits EMT in hepatocellular carcinoma $[26,11]$.

The PI3K/AKT signaling pathway has been proven up-regulated in cancer initiation and invasion [16] and our results demonstrated that miR-203a up-regulation inhibited this pathway. SIX4, also known as AREC3, is part of the sine oculis homeobox (SIX) homolog family [27] and a transcription factor in cell differentiation and development. Therefore, it is involved in cancer progression. For example, SIX4 promotes metastasis in colorectal cancer [28] and correlates with higher TNM stage in non-small cell lung cancer [29]. Consequently, SIX4 was identified herein as both an oncogene and miR-203a target.

Herein, we established that (1) miR-203a is able to suppress the expression of SIX 4 at both the protein and mRNA levels in vitro; (2) the luciferase reporter assay showed that miR-203a directly recognized the 3'-UTR of SIX4 transcripts; (3) the expression of miR-203a was negatively correlated with the levels of SIX4 in vivo; (4) Knock-down of SIX4 mimicked the effects of over-expression of miR-203a in bladder cancer cells, while the over-expression of SIX 4 reversed these effects.

In conclusion, our study identified miR-203a as a tumor suppressor in human bladder cancer cells, where it acts at least partly by inhibiting SIX4. Expression of miR-203a/SIX4 may therefore be useful as a prognostic factor in bladder cancer, but its therapeutic value in cancer progression requires robust trials.

\section{References}

[1] SIEGEL RL, MILLER KD, JEMAL A. Cancer statistics, 2016. CA Cancer J Clin 2016; 66: 7-30. https://doi.org/10.3322/ caac. 21332

[2] CANCER GENOME ATLAS RESEARCH NETWORK. Comprehensive molecular characterization of urothelial bladder carcinoma. Nature 2014; 507: 315-322. https://doi. org/10.1038/nature12965

[3] KNOWLES MA, HURST CD. Molecular biology of bladder cancer: new insights into pathogenesis and clinical diversity. Nat Rev Cancer 2015; 15: 25-41. https://doi.org/10.1038/ nrc3817

[4] BARTEL DP. MicroRNAs: target recognition and regulatory functions. Cell 2009; 136: 215-233. https://doi.org/10.1016/j. cell.2009.01.002

[5] SHUKLA GC, SINGH J, BARIK S. MicroRNAs: Processing, Maturation, Target Recognition and Regulatory Functions. Mol Cell Pharmacol 2011; 3: 83-92

[6] DAVIS-DUSENBERY BN, HATA A. MicroRNA in Cancer: The Involvement of Aberrant MicroRNA Biogenesis Regulatory Pathways. Genes Cancer 2010; 1: 1100-1114. https:// doi.org/10.1177/1947601910396213

[7] LIN S, GREGORY RI. MicroRNA biogenesis pathways in cancer. Nat Rev Cancer 2015; 15: 321-333. https://doi. org/10.1038/nrc3932 
[8] XIU Y, LIU Z, XIA S, JIN C, YIN H et al. MicroRNA-137 upregulation increases bladder cancer cell proliferation and invasion by targeting PAQR3. PLoS One 2014; 9: e109734. https://doi.org/10.1371/journal.pone.0109734

[9] YAN L, WANG Y, LIANG J, LIU Z, SUN X et al. MiR-301b promotes the proliferation, mobility, and epithelial-tomesenchymal transition of bladder cancer cells by targeting EGR1. Biochem Cell Biol 2017; 95: 571-577. https://doi. org/10.1139/bcb-2016-0232

[10] SHANG A, YANG M, SHEN F, WANG J, WEI J et al. MiR1-3p Suppresses the Proliferation, Invasion and Migration of Bladder Cancer Cells by Up-Regulating SFRP1 Expression. Cell Physiol Biochem 2017; 41: 1179-1188. https://doi. org/10.1159/000464379

[11] WANG L, TONG D, GUO Q, WANG X, WU F et al. HOXD3 targeted by miR-203a suppresses cell metastasis and angiogenesis through VEGFR in human hepatocellular carcinoma cells. Sci Rep 2018; 8: 2431. https://doi.org/10.1038/s41598018-20859-3

[12] WANG L, SUN H, WANG X, HOU N, ZHAO L et al. EGR1 mediates miR-203a suppress the hepatocellular carcinoma cells progression by targeting HOXD3 through EGFR signaling pathway. Oncotarget 2016; 7: 45302-45316. https://doi. org/10.18632/oncotarget.9605

[13] JIANG N, JIANG X, CHEN Z, SONG X, WU L et al. MiR203a-3p suppresses cell proliferation and metastasis through inhibiting LASP1 in nasopharyngeal carcinoma. J Exp Clin Cancer Res 2017; 36: 138. https://doi.org/10.1186/s13046017-0604-3

[14] SHEN J, ZHANG J, XIAO M, YANG J, ZHANG N. MiR-203 suppresses bladder cancer cell growth and targets the Twist 1 . Oncol Res 2018; 26: 1155-1165. https://doi.org/10.3727/096 504017X15041934685237

[15] ZHANG X, ZHANG Y, LIU X, FANG A, LI P et al. MicroRNA-203 Is a Prognostic Indicator in Bladder Cancer and Enhances Chemosensitivity to Cisplatin via Apoptosis by Targeting Bcl-w and Survivin. PLoS One 2017; 10: e0143441. https://doi.org/10.1371/journal.pone.0143441

[16] YU R, YU BX, CHEN JF, LV XY, YAN ZJ et al. Anti-tumor effects of Atractylenolide I on bladder cancer cells. J Exp Clin Cancer Res 2016; 35: 40. https://doi.org/10.1186/s13046016-0312-4

[17] HSU YL, KUO YC, KUO PL, NG LT, KUO YH et al. Apoptotic effects of extract from Antrodia camphorata fruiting bodies in human hepatocellular carcinoma cell lines. Cancer Lett 2005; 221: 77-89. https://doi.org/10.1016/j.canlet.2004.08.012

[18] XU M, LI J, WANG X, MENG S, SHEN J et al. MiR-22 suppresses epithelial-mesenchymal transition in bladder cancer by inhibiting Snail and MAPK1/Slug/vimentin feedback loop. Cell Death Dis 2018; 9: 209. https://doi.org/10.1038/ s41419-017-0206-1
[19] YANG D, DU G, XU A, XI X, LI D. Expression of miR-149-3p inhibits proliferation, migration, and invasion of bladder cancer by targeting S100A4. Am J Cancer Res 2017; 7: 2209-2219.

[20] FENG C, SUN P, HU J, FENG H, LI M et al. miRNA-556$3 p$ promotes human bladder cancer proliferation, migration and invasion by negatively regulating DAB2IP expression. Int J Oncol 2017; 50: 2101-2112. https://doi.org/10.3892/ ijo.2017.3969

[21] LI Y, SHAN Z, LIU C, YANG D, WU J et al. MicroRNA-294 Promotes Cellular Proliferation and Motility through the PI3K/AKT and JAK/STAT Pathways by Upregulation of NRAS in Bladder Cancer. Biochemistry (Mosc) 2017; 82: 474-482. https://doi.org/10.1134/S0006297917040095

[22] LIU W, DONG Z, LIANG J, GUO X, GUO Y et al. Downregulation of Potential Tumor Suppressor miR-203a by Promoter Methylation Contributes to the Invasiveness of Gastric Cardia Adenocarcinoma. Cancer Invest 2016; 34: 506-516. https://doi.org/10.1080/07357907.2016.1242010

[23] GOMES BC, MARTINS M, LOPES P, MORUJAO I, OLIVEIRA M et al. Prognostic value of microRNA-203a expression in breast cancer. Oncol Rep 2016; 36: 1748-1756. https://doi. org/10.3892/or.2016.4913

[24] HU G, LAI P, LIU M, XU L, GUO Z et al. miR-203a regulates proliferation, migration, and apoptosis by targeting glycogen synthase kinase-3beta in human renal cell carcinoma. Tumour Biol 2014; 35: 11443-11453. https://doi.org/10.1007/ s13277-014-2476-X

[25] YANG CH, WANG Y, SIMS M, CAI C, HE P et al. MicroRNA203a suppresses glioma tumorigenesis through an ATMdependent interferon response pathway. Oncotarget 2017; 8: 112980-112991. https://doi.org/10.18632/oncotarget.22945

[26] LIU D, WU J, LIU M, YIN H, HE J et al. Downregulation of miRNA-30c and miR-203a is associated with hepatitis C virus core protein-induced epithelial-mesenchymal transition in normal hepatocytes and hepatocellular carcinoma cells. Biochem Biophys Res Commun 2015; 464: 1215-1221. https://doi.org/10.1016/j.bbrc.2015.07.107

[27] SEOHC,CURTISS J, MLODZIKM,FJOSE A. Six class homeoboxgenesin drosophilabelong to three distinct families and are involved in head development. Mech Dev 1999; 83: 127-139.

[28] LI G, HU F, LUO X, HU J, FENG Y. SIX4 promotes metastasis via activation of the PI3K-AKT pathway in colorectal cancer. PeerJ 2017; 5: e3394. https://doi.org/10.7717/peerj.3394

[29] LIU Q, LI A, TIAN Y, LIU Y, LI T et al. The expression profile and clinic significance of the SIX family in non-small cell lung cancer. J Hematol Oncol 2016; 9: 119. https://doi. org/10.1186/s13045-016-0339-1 